\title{
COMT-Catalyzed Palmitic Acid Methyl Ester Biosynthesis in Perivascular Adipose Tissue and its Potential Role Against Hypertension $₫$
}

\author{
(1) Chin-Hung Liu, Hao-Jen Hsu, Tzu-Ling Tseng, Tsung-Jen Lin, Wei-Hsiang Weng, \\ Mei-Fang Chen, ${ }^{1}$ and Tony Jer-Fu Lee ${ }^{1}$
}

Department of Pharmacology, College of Medicine (C.-H.L., T.-J.L.), Department of Life Sciences, College of Medicine (H.-J.H., W.-H.W.), and Ph.D program in Pharmacology and Toxicology, Department of Medicine, School of Medicine (C.-H.L., T.-J.L.), Tzu Chi University, Hualien, Taiwan; Department of Medical Research (T.-L.T., M.-F.C., T.J.-F.L.) and Cardiovascular Research Center (C.-H.L., T.-L.T., M.-F.C., T.J.-F.L.), Hualien Tzu Chi Hospital, Buddhist Tzu Chi Medical Foundation, Hualien, Taiwan; and Department of Pharmacology, Southern Illinois University School of Medicine, Springfield, Illinois (T.J.-F.L.)

Received October 31, 2019; accepted February 10, 2020

\section{ABSTRACT}

Decreased release of palmitic acid methyl ester (PAME), a vasodilator, from perivascular adipose tissue (PVAT) might contribute to hypertension pathogenesis. However, the PAME biosynthetic pathway remains unclear. In this study, we hypothesized that PAME is biosynthesized from palmitic acid (PA) via human catechol-O-methyltransferase (COMT) catalysis and that decreased PAME biosynthesis plays a role in hypertension pathogenesis. We compared PAME biosynthesis between agematched normotensive Wistar Kyoto (WKY) rats and hypertensive spontaneously hypertensive rats (SHRs) and investigated the effects of losartan treatment on PAME biosynthesis. Computational molecular modeling indicated that PA binds well at the active site of COMT. Furthermore, in in vitro enzymatic assays in the presence of COMT and $S-5^{\prime}$-adenosyl- $L$-methionine (AdoMet), the stable isotope $\left[{ }^{13} \mathrm{C}_{16}\right]$-PA was methylated to form $\left[{ }^{13} \mathrm{C}_{16}\right]$ PAME in incubation medium or the Krebs-Henseleit solution containing 3T3-L1 adipocytes or rat PVAT. The adipocytes and PVATs expressed membrane-bound (MB)-COMT and soluble (S)COMT proteins. $\left[{ }^{13} \mathrm{C}_{16}\right]$-PA methylation to form $\left[{ }^{13} \mathrm{C}_{16}\right]$-PAME in 3T3-L1 adipocytes and rat PVAT was blocked by various COMT inhibitors, such as $S$-(5'-adenosyl)- $L$-homocysteine, adenosine$2^{\prime}, 3^{\prime}$-dialdehyde, and tolcapone. MB- and S-COMT levels in PVATs of established SHRs were significantly lower than those in PVATs of age-matched normotensive WKY rats, with decreased $\left[{ }^{13} \mathrm{C}_{16}\right]$-PA methylation to form $\left[{ }^{13} \mathrm{C}_{16}\right]$-PAME. This decrease was reversed by losartan, an angiotensin II (Ang II) type 1 receptor antagonist. Therefore, PAME biosynthesis in rat PVAT is dependent on AdoMet, catalyzed by COMT, and decreased in SHRs, further supporting the role of PVAT/PAME in hypertension pathogenesis. Moreover, the antihypertensive effect of losartan might be due partly to its increased PAME biosynthesis.

\section{SIGNIFICANCE STATEMENT}

PAME is a key PVAT-derived relaxing factor. We for the first time demonstrate that PAME is synthesized through PA methylation via the $S-5$ '-adenosyl- $L$-methionine-dependent COMT catalyzation pathway. Moreover, we confirmed PVAT dysfunction in the hypertensive state. COMT-dependent PAME biosynthesis is involved in Ang II receptor type 1-mediated blood pressure regulation, as evidenced by the reversal of decreased PAME biosynthesis in PVAT by losartan in hypertensive rats. This finding might help in developing novel therapeutic or preventive strategies against hypertension.
This work was supported by grants from Tzu Chi Medical Foundation [TCMMP104-01-04], National Health Research Institutes [NHRI-EX10410232SI], and the Ministry of Science and Technology [MOST105-2320-B303-001-MY3 and MOST106-2320-B-320-011-] of Taiwan.

${ }^{1}$ M.-F.C. and T.J.-F.L. contributed equally to this work.

Tony Jer-Fu Lee died on February 15th, 2018.

https://doi.org/10.1124/jpet.119.263517.

S This article has supplemental material available at jpet.aspetjournals.org.

\section{Introduction}

The significant roles of PVAT in regulating the vascular tone (Lee et al., 2011; Eringa et al., 2012) and vascular homeostasis (Chang et al., 2012; Szasz et al., 2013) are well recognized. PVATs of different vascular beds release both dilative and constrictive factors according to the body's need. Among these factors, PVAT-derived relaxing factors (PVATRFs) have attracted much interest (Tano et al., 2014). Several

ABBREVIATIONS: $\Delta G_{\text {bind, }}$, binding-free energy; 3H4MAP, 3'-hydroxy-4'-methoxyacetophenone; 4H3MAP, 4'-hydroxy-3'-methoxyacetophenone; Ado, adenosine; AdoHcy, S-(5'-adenosyl)-L-homocysteine; AdoMet, $S$-5'-adenosyl-L-methionine; Adox, adenosine-2' $5^{\prime} 3^{\prime}$-dialdehyde; Ang II, angiotensin II; apo-human S-COMT, a human S-COMT without ligand binding; AT-1, Ang II receptor type 1; COMT, catechol-Omethyltransferase; DHAP, 3,4-dihydroxyaceto-phenone; DNC, 3,5-dinitrocatechol; FAME, fatty acid methyl ester; GC-MS, gas chromatography-mass spectrometry; MB, membrane bound; MD, molecular dynamics; PA, palmitic acid; PAME, palmitic acid methyl ester; PVAT, perivascular adipose tissue; PVATRF, PVAT-derived relaxing factor; S, soluble; SD, Sprague-Dawley; SHR, spontaneously hypertensive rat; TG, triacylglycerol; WKY, Wistar Kyoto. 
substances, such as adiponectin (Fésüs et al., 2007; Xu et al., 2010), hydrogen sulfide (Wójcicka et al., 2011), nitric oxide (Gao et al., 2007), and prostaglandin $\mathrm{I}_{2}$, are considered as a PVATRF. PAME, a novel vasodilator that attenuates the constrictor-mediated active response is released from PVAT and is also considered as a PVATRF (Lee et al., 2011).

In SHR animals, PAME amount released from PVAT and PVAT-related relaxation decrease in aorta, which can be reversed by losartan, an antihypertensive drug (Lee et al., 2011). PAME levels in PVAT might play a role in hypertension pathogenesis. In addition, PAME might enhance cerebral blood flow, alleviate neuronal cell death, and improve cognitive functions in animals subjected to asphyxial cardiac arrest (Lee et al., 2019). Although the evidences for physiologic roles of PAME are emerging, the PAME biosynthesis in PVAT remains unknown.

COMT (EC 2.1.1.6) is an AdoMet-dependent enzyme (Männistö and Kaakkola, 1999). The main function of COMT is to degrade various catecholamines (e.g., dopamine, epinephrine, and norepinephine) (Hirano et al., 2007) and catecholestrogens (e.g., 2-hydroxyestradiol) (Hernandez et al., 2013) via $O$-methylation. The binding for AdoMet, $\mathrm{Mg}^{2+}$, and catecholsubstrate are strictly controlled in a catalytic circle (Ma et al., 2014). After AdoMet, $\mathrm{Mg}^{2+}$, and substrate bind to COMT, the methyl group attached to the methionine sulfur atom of AdoMet is oriented toward the substrate-binding site for methylation (Ma et al., 2014). Most tissues, including adipose, contain two forms of COMT, membrane-bound (MB) and soluble (S) forms (Borchardt and Huber, 1975; Song et al., 2019).

Changes in COMT activity or expression have been implicated in hypertension and heart failure (Houston, 2007). The systolic blood pressure is significantly increased in $C O M T^{-1-}$ mice (Kanasaki et al., 2008; Stanley et al., 2012). The human genotypic variant of functional COMT with valine(158)methionine is related to hypertension risk (Stewart et al., 2009; Htun et al., 2011). Previous studies have demonstrated decreased MB-form COMT activity and protein expression in the liver (Tsunoda et al., 2003), brain (Masuda et al., 2006), and renal cortex (Ooshima et al., 2009) of SHRs compared with those of age-matched normotensive WKY rats.

In this study, we hypothesized that PAME is biosynthesized from PA via COMT catalysis and that the decreased PAME biosynthesis is involved in hypertension pathogenesis. We compared PAME biosynthesis between agematched normotensive WKY rats and hypertensive SHRs and investigated the effect of losartan treatment on PAME biosynthesis.

\section{Materials and Methods}

\section{Animals and Aortic PVAT Sampling}

Adult male Sprague-Dawley (SD) rats (body weight, 350-400 g), 8and 13-week-old male SHRs, and 8- and 13-week-old male WKY rats (BioLASCO Co., Ltd., Taipei, Taiwan) were housed in the animal quarters at Tzu Chi University, Taiwan, under a 12-hour light/dark cycle. All rats were fed a standard ration and provide tap water ad libitum. The rats were anesthetized using penotobarbital $(50 \mathrm{mg} / \mathrm{kg}$, i.p.), and PVAT surrounding the thoracic aorta was quickly excised and processed for PAME biosynthesis, COMT protein expression, and immunocytochemistry studies.
This study was performed in accordance with the ethical principles of animal research, and the animal protocol was approved by the ethics committee of Tzu Chi University.

\section{Measurement of the Arterial Blood Pressure}

Systolic blood pressure was measured by the tail-cuff method using a programmed MK-2000ST electro sphygmomanometer (Muromachi, Tokyo, Japan) as previously described (Chou et al., 2013). Table 1 shows systolic blood pressure data.

\section{Chemicals, Substrates, and Reagents}

AdoMet, $S$-(5'-adenosyl)- $L$-homocysteine (AdoHcy), adenosine- $2^{\prime}, 3^{\prime}$-dialdehyde (Adox), COMT, $\left[{ }^{13} \mathrm{C}_{16}\right]$-PA, tolcapone, 3,4-dihydroxyacetophenone (DHAP), 3'-hydroxy-4' -methoxyacetophenone (3H4MAP), 4' -hydroxy3'-methoxyacetophenone (4H3MAP), N-tris(hydroxymethyl)-methyl2-aminoethane sulphonic acid, methyl pentadecanoate, PAME, and losartan were purchased from Sigma-Aldrich (St. Louis, MO). Ethyl acetate, ethanol, and hexane were purchased from Mallinckrodt (St. Louis, MO).

\section{Computational Modeling Analysis of Human S-COMT}

Molecular Docking. First, three ligands DHAP (a standard ligand for COMT), 3,5-dinitrocatechol (DNC; a competitive COMT inhibitor), and PA selected for molecular docking were constructed using MOE2013.08 (Molecular Operating Environment, http://www. chemcomp.com). Ligand topologies for subsequent simulations were obtained from the GlycoBioChem PRODRG2 web server (http:// davapc1.bioch.dundee.ac.uk/cgi-bin/prodrg), provided by Professor Daan van Aalten (Schüttelkopf and van Aalten, 2004) under the GROMOS 53A6 force field, which is also suitable for biomolecules. The apo-human S-COMT (Protein Data Bank code 3A7E), which is a COMT protein lacking DNC ligand (only COMT, AodMet, and $\mathrm{Mg}^{2+}$ ), was set as a receptor for binding of various ligands. The initial favorable sites for the binding of each ligand to apo-S-COMT were determined using the docking module of MOE2013.08.

Molecular Dynamics Simulations. The DNC-apo-S-COMT complex was placed in an $80 \times 80 \times 80 \AA$ water box containing more than 10,000 water molecules. To neutralize DNC-apo-S-COMT binding, $48 \mathrm{Na}^{+}$and $43 \mathrm{Cl}^{-}$, forming a $100 \mathrm{mM} \mathrm{NaCl}$ solution, were added following energy minimization. A total of 45,672 atoms of the complex consisted of 2090 atoms of COMT, 36 atoms of AdoMet, 18 atoms of DNC, $1 \mathrm{Mg}^{2+}$, and 14,479 simple point-charge water molecules, including $48 \mathrm{Na}^{+}$and $43 \mathrm{Cl}^{-}$. All molecular dynamics (MD) simulations were run for $200 \mathrm{~ns}$ to calculate the binding-free energy (Table 2). Detailed MD simulation methods and equilibration protocols have been previously described (Liou et al., 2014).

Molecular Mechanics Poisson-Boltzmann Surface Area Binding-Free Energy Calculations. To define the most stable complexes predicted by molecular docking, the Molecular Mechanics Poisson-Boltzmann Surface Area approach was used for estimating the binding-free energy $\left(\Delta G_{\text {bind }}\right)$ on the basis of snapshots extracted from a single trajectory of the DNC-apo-S-COMT complex (singletrajectory method) (Hou et al., 2011a,b). The single-trajectory method is much faster and requires less sampling than the individual trajectory method, which requires three separate MD simulations on system components (receptor, ligand, and complex) (Hou and $\mathrm{Yu}$, 2007; Xu et al., 2013). We assumed that the receptor and ligand behaved similarly during binding, which was reasonable and adoptable for our simulations. The free energy calculations have been explained in detail previously (Jiang et al., 2015; Chang et al., 2017). To calculate all energy terms, we extracted 300 snapshots from 30 (170-200)-ns molecular docking trajectories per system.

In Vitro Enzymatic Assay of COMT-Catalyzed Biosynthesis from PA. We assayed COMT activity in vitro as described by Tehlivets (2011). Briefly, we placed glass tubes on ice. To evaluate the $O$-methylation of DHAP dissolved in deionized water (positive 
TABLE 1

Body weight and systolic blood pressure of SHRs and WKY rats

Values are presented as means \pm S.E.M.

\begin{tabular}{llll}
\hline Age (wk) & Strain $(n)$ & Body weight $(\mathrm{g})$ & SBP $(\mathrm{mm} \mathrm{Hg})$ \\
\hline 8 & WKY (15) & $243.3 \pm 28.9$ & $114.0 \pm 2.6$ \\
& SHR (15) & $250.0 \pm 13.2$ & $128.1 \pm 10.0$ \\
13 & WKY (7) & $321.7 \pm 14.4$ & $121.5 \pm 4.2$ \\
& SHR (7) & $290.0 \pm 10.0^{*}$ & $182.1 \pm 13.3^{*}$ \\
\hline
\end{tabular}

SBP, systolic blood pressure.

$* P<0.05$ vs. age-matched WKY rats.

control) or $\left[{ }^{13} \mathrm{C}_{16}\right]$-PA dissolved in absolute ethanol, we incubated $100 \mu \mathrm{M}$ of either substrate with COMT enzyme extracted from porcine liver (C1897; Sigma-Aldrich) of 10-30 U from a stock solution of 1000 $\mathrm{U} / \mathrm{ml}$ of COMT in cold N-tris(hydroxymethyl)-methyl-2-aminoethane sulphonic acid diluent buffer at $\mathrm{pH} 7.6$ in the presence of $1.2 \mathrm{mM}$ $\mathrm{MgCl}_{2}$ and $4 \mathrm{mM}$ dithiothreitol. The reaction was initiated by adding $1 \mathrm{mM}$ AdoMet for 1 hour at $37^{\circ} \mathrm{C}$ and then terminated by adding $0.2 \mathrm{M}$ sodium borate ( $\mathrm{pH} 10.0)$. The reaction products were analyzed for methylated DHAP (3H4MAP and 4H3MAP) or $\left[{ }^{13} \mathrm{C}_{16}\right]$-PAME using a spectrophotometer and gas chromatography-mass spectrometry (GC-MS), respectively; absorbance was measured at $344 \mathrm{~nm}$. Mediums containing no AdoMet (blank) or COMT enzyme served as negative controls. The assay was linear over a range of concentrations from 1 to $100 \mu \mathrm{M}$, with a correlation coefficient of $r^{2}=0.9998(P<0.0001)$ for $O$-methylated DHAP and $0.9972(P<0.0001)$ for PAME.

GC-MS. Reaction samples from in vitro enzymatic assays, condition mediums, and Krebs-Henseleit solution from incubation with 3T3-L1 adipocytes and isolated PVAT preparations were added with $10 \mu \mathrm{M}$ pentadecanoic acid methyl ester, which acts as internal standard. Next, the samples were lyophilized and resolved using ethyl acetate to further dissolve the organic compounds as previously described (Lin et al., 2008; Lee et al., 2011). After vortexing, sonicating, and centrifuging at $1500 \mathrm{rpm}$ for 5 minutes at $4^{\circ} \mathrm{C}$, the supernatant was transferred to a glass sample vial to form a $2-\mu l$ splitless injection. The samples were detected using a HewlettPackard Model 6890 GC system (Hewlett-Packard, Palo Alto, CA) assembled with a G1512A autosampler and interfaced to a 5973 mass selective detector. An HP-5MS 5\% phenyl polysilphenylene-siloxane capillary column $(10 \mathrm{~m} \times 0.32 \mathrm{~mm}$ i.d.; film thickness $0.5 \mu \mathrm{m})$ was used; helium gas was used as the carrier gas at a flow rate of $0.7 \mathrm{ml} /$ min. The temperatures of the GC injection port and interface were maintained at $250^{\circ} \mathrm{C}$ and $300^{\circ} \mathrm{C}$, respectively. The starting temperature of GC was set at $90^{\circ} \mathrm{C}$, which was increased to $240^{\circ} \mathrm{C}$ at $15^{\circ} \mathrm{C} / \mathrm{min}$ and then increased up to $300^{\circ} \mathrm{C}$ at $10^{\circ} \mathrm{C} / \mathrm{min}$. Mass spectrum scanning was set at $50-500 \mathrm{~m} / \mathrm{z}$, and the electron ionization energy of the electron impact mode was set at $70 \mathrm{eV}$. Hewlett-Packard G1701AA version 0.300 ChemStation Software was used for data acquisition and analysis in the drug analysis mode. $O$-methylated PAME $\left(\mathrm{M}^{+}\right.$, $270 \mathrm{~m} / z)$ and $\left[{ }^{13} \mathrm{C}_{16}\right]$-PAME $\left(\mathrm{M}^{+}, 286 \mathrm{~m} / z\right)$ were analyzed with single diagnostic ion-based GC-MS (Supplemental Fig. 1) to qualitatively and quantitatively analyze PAME and $\left[{ }^{13} \mathrm{C}_{16}\right]$-PAME, as described previously (Lin et al., 2008; Lee et al., 2010, 2011).
3T3-L1 Adipocyte Culture and Differentiation. Mouse embryo preadipocyte (fibroblasts) 3T3-L1 cells were obtained from the American Type Culture Collection and cultured in 12-well cluster plates in Dulbecco's Modified Eagle's Medium (HyClone, Logan, UT) supplemented with $10 \%$ calf serum (Gibco, Grand Island, NY) and $1 \%$ antibiotics (Invitrogen, Carlsbad, CA) until confluence, as described previously (Lee et al., 2011). Two days after confluence (day 0), the fibroblasts were exposed to a differentiation medium comprising $0.5 \mathrm{mM}$ isobutylmethylxanthine, $1 \mu \mathrm{M}$ dexamethasone, $1.67 \mathrm{mM}$ insulin (MDI; Sigma-Aldrich), and 10\% fetal bovine serum for 3 days. Finally, the differentiated 3T3-L1 adipocytes were transferred to Dulbecco's Modified Eagle's Medium with $1.67 \mathrm{mM}$ insulin and $10 \%$ fetal bovine serum and refed every 2 days. Mature 3T3-L1 adipocytes were confirmed using Oil Red O staining of lipid droplets in adipocytes (Supplemental Fig. 2).

Oil Red O Staining. Lipid droplets in mature 3T3-L1 adipocytes were stained with Oil Red O (Supplemental Fig. 2) as described previously with some modifications (Kawai et al., 2007). Briefly, 3T3$\mathrm{L} 1$ adipocytes were fixed with $4 \%$ formaldehyde-phosphate buffer $(\mathrm{pH}$ 7.4) for 1 hour, rinsed with water, and stained with $0.3 \%$ filtered Oil Red O (Sigma-Aldrich) in $100 \%$ isopropanol for 1 hour at $60^{\circ} \mathrm{C}$. Then, the cells were washed twice with distilled water to remove excess dye and photographed under a Leica light microscope (Leica Microsystems, Wetzlar, Germany).

PAME Biosynthesis in 3T3-L1/PVAT Adipocytes and Effects of COMT Inhibitors. 3T3-L1 adipocytes were incubated in 12-well cluster with different concentrations of $\left[{ }^{13} \mathrm{C}_{16}\right]-\mathrm{PA}(100-500 \mu \mathrm{M})$ dissolved in absolute ethanol in $1 \mathrm{ml}$ culture medium ( 24 and 72 hours) in a $5 \% \mathrm{CO}_{2}$ incubator at $37^{\circ} \mathrm{C}$. Culture medium containing $500 \mu \mathrm{M}$ $\left[{ }^{13} \mathrm{C}_{16}\right]$-PA without 3T3-L1 adipocytes was used as a control. AdoHcy, a COMT-catalyzed AdoMet metabolite, inhibits AdoMet-dependent methyltransferases via the feedback inhibitory mechanism (Yeh et al., 1991). Therefore, we determined whether AdoHcy inhibits PAME biosynthesis in 3T3-L1 adipocytes. Different concentrations (100 and $300 \mu \mathrm{M})$ of AdoHcy in dimethyl sulfoxide were added to incubation mediums containing $250 \mu \mathrm{M}\left[{ }^{13} \mathrm{C}_{16}\right]-\mathrm{PA}$ and 3T3-L1 adipocytes. Culture medium containing $250 \mu \mathrm{M}\left[{ }^{13} \mathrm{C}_{16}\right]-\mathrm{PA}$ without $3 \mathrm{~T} 3-\mathrm{L} 1$ adipocytes was used as a negative control, and cells treated with an equivalent amount of $\left[{ }^{13} \mathrm{C}_{16}\right]-\mathrm{PA}$ and dimethyl sulfoxide $(<0.1 \%$; solvent for dissolving AdoHcy) were used as a positive control. We used similar procedures to examine the effects of other COMT inhibitors on PAME biosynthesis, including 10 and $100 \mu \mathrm{M}$ Adox (an

TABLE 2

Free energy values of PA, DHAP, and DNC

Free energy values for the interactions of DHAP, DNC, or PA with S-COMT were calculated by using MM/PBSA approach.

\begin{tabular}{|c|c|c|c|c|c|}
\hline \multirow{2}{*}{ Ligands } & \multirow{2}{*}{$\Delta G_{\mathrm{bind}}(\mathrm{kcal} / \mathrm{mol})$} & \multicolumn{2}{|c|}{$E_{\mathrm{MM}}(\mathrm{kcal} / \mathrm{mol})$} & \multicolumn{2}{|c|}{$G_{\text {solv }}(\mathrm{kcal} / \mathrm{mol})$} \\
\hline & & Coul & vdW & PolSol & NpoSol \\
\hline DHAP & $122.77 \pm 13.23$ & $507.82 \pm 18.75$ & $33.53 \pm 5.30$ & $-420.66 \pm 21.10$ & $2.18 \pm 0.13$ \\
\hline DNC & $-61.49 \pm 14.64$ & $258.12 \pm 30.42$ & $10.78 \pm 6.60$ & $-332.25 \pm 23.18$ & $1.85 \pm 0.17$ \\
\hline $\mathrm{PA}$ & $-40.97 \pm 20.37$ & $474.77 \pm 45.32$ & $17.94 \pm 9.66$ & $-534.93 \pm 30.28$ & $1.26 \pm 0.37$ \\
\hline
\end{tabular}

Coul, Coulomb energy model; $E_{\mathrm{MM}}$, molecular mechanics energy; $G_{\text {solv, solvation-free energy; MM/PBSA, Molecular Mechanics }}$ Poisson-Boltzmann Surface Area; NpoSol, norpolar solvation energy model; PolSol, polar solvation energy model; vdW, van der Waals energy model. 
inhibitor for AdoHcy hydrolase/EC 3.3.1.1) in dimethyl sulfoxide and 1-1000 $\mathrm{nM}$ tolcapone (a highly selective COMT inhibitor) in dimethyl sulfoxide (Vieira-Coelho and Soares-da-Silva, 1999). By inhibiting AdoHcy hydrolase, which catalyzes AdoHcy hydrolysis to form adenosine and homocysteine (Bartel and Borchardt, 1984), Adox acts as an indirect AdoMet-dependent MTase inhibitor by increasing AdoHcy concentration (Kurkela et al., 2004). Finally, all culture mediums were collected and processed for GC-MS analysis.

To determine PAME biosynthesis in rat thoracic aortic PVAT (350-400 g SD, 8- and 13-week-old SHRs or WKY rats), isolated PVAT was incubated in $10 \mathrm{ml}$ Krebs-Henseleit solution with $250 \mu \mathrm{M}$ $\left[{ }^{13} \mathrm{C}_{16}\right]$-PA dissolved in absolute ethanol for $1-90$ minutes at $37^{\circ} \mathrm{C}$. Krebs-Henseleit solution with $250 \mu \mathrm{M}\left[{ }^{13} \mathrm{C}_{16}\right]$-PA without PVAT was used as a negative control. To determine whether COMT inhibitors (tolcapone) inhibit PAME biosynthesis in rat thoracic aortic PVAT, isolated PVAT was preincubated in $10 \mathrm{ml}$ Krebs-Henseleit solution containing different concentrations of tolcapone ( $300 \mathrm{nM}$ and $1 \mu \mathrm{M})$ for 30 minutes at $37^{\circ} \mathrm{C}$. Preincubated PVAT was then transferred to a different tissue bath containing $10 \mathrm{ml}$ Krebs-Henseleit solution and $250 \mu \mathrm{M}\left[{ }^{13} \mathrm{C}_{16}\right]$-PA dissolved in absolute ethanol and incubated for 1-90 minutes at $37^{\circ} \mathrm{C}$. In addition, isolated PVAT preparations (from 13 -week-old SHRs or WKY rats) were incubated in $10 \mathrm{ml}$ KrebsHenseleit solution containing $1 \mu \mathrm{M}$ losartan in dimethyl sulfoxide alone or $1 \mu \mathrm{M}$ losartan plus tolcapone in dimethyl sulfoxide for 30 minutes. Krebs-Henseleit solution containing $250 \mu \mathrm{M}\left[{ }^{13} \mathrm{C}_{16}\right]-\mathrm{PA}$ without PVAT was used as a negative control. All samples were collected and processed for GC-MS assay. Cumulative $\left[{ }^{13} \mathrm{C}_{16}\right]$-PAME concentrations from different incubation periods were calculated from the area under the curve.

Western Blots. Bicinchoninic acid assay (Pierce Chemicals, Rockford, IL) was used for protein quantification. Cell/tissue extracts were diluted by adjunction of four parts of sample buffer $(0.25 \mathrm{M}$ Tris-HCl, pH 6.8; $2 \%$ 2-mecaptoethanol, $8 \%$ sodium dodecyl sulfate, $0.02 \%$ bromophenol blue, and $40 \%$ glycerol) and boiled for 5 minutes at $100^{\circ} \mathrm{C}$. Protein extracts were quantified. Then, $20 \mathrm{mg}$ of each protein was separated on $10 \%$ SDS polyacrylamide gel and transferred onto polyvinylidene difluoride membranes (Bio-Rad, Hercules, CA) using semidry electroblotting (Amersham Biosciences, Buckinghamshire, UK). Polyvinylidene difluoride membranes were blocked with $5 \%$ nonfat milk/0.25\% Tris-buffered saline containing Tween 20 for 2 hours at room temperature (RT). The mouse COMT monoclonal immunoglobulin G (IgG) antibody (1:2000; BD Transduction, CA), antiactin antibody (1:4000; Chemicon, IL), or antitubulin antibody (1:4000; Chemicon) were incubated overnight at $4^{\circ} \mathrm{C}$, and horseradish peroxidase-conjugated secondary antibodies (1:2000; KPL, Washington, DC) were added for 1 hour at RT. Protein expression was detected using a Biospectrum 810 UVP system (Thermo Fisher Scientific, Waltham, MA) with enhanced chemiluminescence reagent (PerkinElmer Life Science, MA). ImageJ version 1.51 (National Institutes of Health, Bethesda, MD) was used for proteins analysis (Tseng et al., 2012).

COMT Immunofluorescence Staining in Rat Aortic PVAT. Standard immunofluorescence staining techniques were used to demonstrate COMT immunoreactivities in 8- and 13-week-old SHR or WKY rat aortic PVAT (Tseng et al., 2016). Briefly, $2-\mu \mathrm{m}$-thick sections of paraffin-embedded PVAT were processed through antigen retrieval buffer and permeabilized with Triton X-100 for 30 minutes. Their nonspecific interaction with antibodies was blocked by incubation with normal serum (BiogeneX) for 1 hour. After washed three times with buffer, the samples were allowed to interact with purified anti-COMT mouse antibody (1:200; BD Bioscience, Franklin Lakes, NJ) overnight at $4^{\circ} \mathrm{C}$ and antimouse IgG labeled with Hilyte Fluor 555 (1:200; AnaSpec) for 2 hours. Cell nuclei were counterstained with 4',6-diamidino-2-phenylindole (DAPI, 1:200; KPL) for 20 minutes. All sections were mounted with a water-soluble mounting media and examined under a Leica fluorescence microscope (Leica Microsystems). A few sections were processed by incubation with primary antibodies but without secondary antibodies serving as a negative control.

Hematoxylin-Eosin $\mathbf{Y}$ Staining. Rat aortas with the intact PVAT surrounding were sectioned and examined by using standard hematoxylin-eosin $\mathrm{Y}$ staining technique as previously described (Tseng et al., 2012). Briefly, 2- $\mu \mathrm{m}$ paraffin-embedded cross-sections were placed on the slides. After deparaffinized with a nonxylene solution (Sigma-Aldrich), the specimens were rehydrated with a serial immersion in ethanol at $100 \%$ for 3 minutes twice, $95 \%$ for 1 minute, and $75 \%$ for 1 minute. Then, the samples were stained with hematoxylin for 3 minutes and subsequently with eosin $\mathrm{Y}$ for 45 seconds. After incubated in nonxylene solution for 6 minutes, the specimens were mounted with coverslips and examined under a light microscope (Olympus, Tokyo, Japan).

Statistical Analysis. Statistical analyses were performed using one-way analysis of variance and Student's $t$ test. Post hoc comparisons between groups were performed using Bonferroni's multiple comparison test. Experimental data are presented as means and S.E.M. All data were analyzed using SPSS version 18.0 (SPSS Inc., Chicago, IL). $P<0.05$ was considered as statistically significant.

\section{Results}

Molecular Docking between PA and S-COMT Using Computational Modeling. Computational molecular modeling analysis demonstrated that PA binds to S-COMT at a specific substrate pocket, which is appropriate for methylation in the presence of AdoMet and $\mathrm{Mg}^{2+}$ (Fig. 1A). The predicted complex structure of DNC/S-COMT by computational molecular modeling was superposed with that of the crystal structure for comparison. The predicted binding position of DNC with S-COMT was at the binding pocket, which is quite similar to that of the crystal structure. By modeling, PA and DHAP bound to the same pocket of S-COMT as DNC did.

The root-mean-square deviations for PA, DHAP, and DNC binding to S-COMT fluctuated from 0.1 to $0.28 \mathrm{~nm}$. This result indicates that $\mathrm{PA}$ can stabilize at the binding pocket of S-COMT similar to DHAP and DNC binding to S-COMT (Fig. 1B).

Table 2 summarizes the free energy levels for PA, DHAP, and DNC binding to S-COMT. $\Delta G_{\text {bind }}$ for DNC binding ( -61.49 $\pm 14.64 \mathrm{kcal} / \mathrm{mol})$ was lower than that for PA $(-40.97 \pm 20.37$ $\mathrm{kcal} / \mathrm{mol})$ and DHAP $(122.77 \pm 13.23 \mathrm{kcal} / \mathrm{mol})$ binding. $\Delta G_{\text {bind }}$ for PA-S-COMT was between that for DHAP-S-COMT and DNC-S-COMT, indicating that the binding affinity of PA is stronger than that of DHAP but weaker than that for DNC.

COMT-Catalyzed PAME Biosynthesis from PA. To determine whether COMT plays a role in PA methylation during PAME biosynthesis, we administered a synthetic stable isotope $\left[{ }^{13} \mathrm{C}_{16}\right]$-PA to distinguish the sources of methylated products. In vitro enzymatic assays showed that $\left[{ }^{13} \mathrm{C}_{16}\right]$-PA was concentration-dependently transformed to $\left[{ }^{13} \mathrm{C}_{16}\right]$-PAME in the presence of COMT and AdoMet. As a positive control, DHAP formed 3H4MAP/4H3MAP after the addition of COMT and AdoMet. However, no methylated products of DHAP or $\left[{ }^{13} \mathrm{C}_{16}\right]$-PA were detected in the absence of COMT or AdoMet (Fig. 2A). The 100 or $500 \mu \mathrm{M}\left[{ }^{13} \mathrm{C}_{16}\right]$-PA were added to culture plates containing preadipocytes (undifferentiated fibroblasts) or 3T3-L1 adipocytes (differentiated). $\left[{ }^{13} \mathrm{C}_{16}\right]$-PAME concentrations in the medium with 3T3-L1 adipocytes were significantly higher than those in the medium with preadipocytes (Fig. 2B). In addition, both MB-COMT and S-COMT were expressed in preadipocytes and 
A

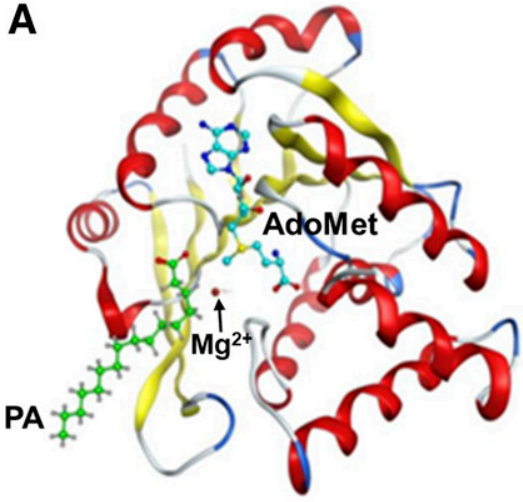

B

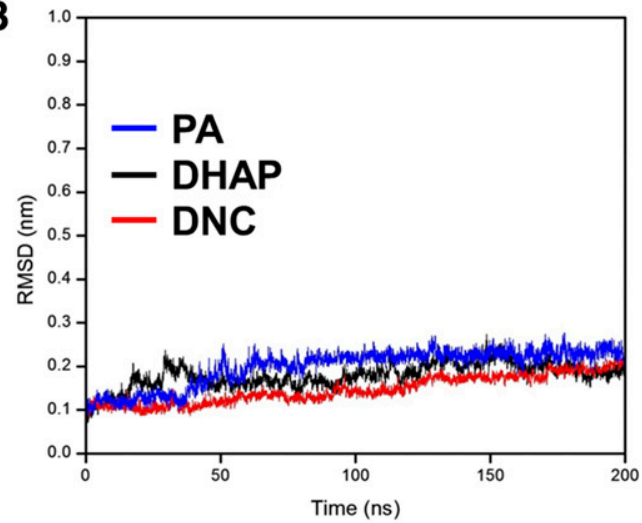

C

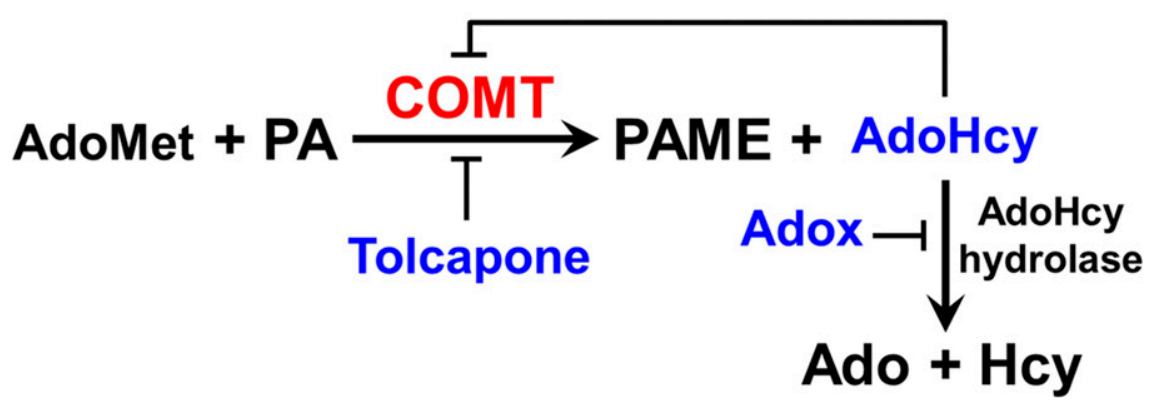

Fig. 1. Computational molecular modeling analysis of S-COMT with PA, DHAP, and DNC. (A) Cartoon representation showing the structure of soluble (S)-COMT adopted from Protein Data Bank (code: 3A7E). The binding pockets for PA, AdoMet, and $\mathrm{Mg}^{2+}$ are indicated. (B) Root-meansquare deviation was plotted as a function of simulation times, representing S-COMT-PA (blue line), S-COMT-DHAP (black line), and S-COMT-DNC (red line) interactions. (C) Hypothetic scheme showing COMTcatalyzed PAME biosynthesis. Tocalpone, AdoHcy, and Adox are inhibitors for the respective enzymes as indicated. Hcy, $L$-homocysteine; Met, $L$-methionine.
3T3-L1 adipocytes, with higher expression for the latter than for the former (Fig. 2, C and D; Supplemental Fig. 4).

Blockade of PAME Biosynthesis by COMT Inhibitors in 3T3-L1 and PVAT Adipocytes. Substrate inhibitors shown in the scheme (Fig. 1C), AdoHcy (100 and $300 \mu \mathrm{M})$ and Adox (10 and $100 \mu \mathrm{M}$ ), suppressed endogenous PAME and $\left[{ }^{13} \mathrm{C}_{16}\right]$-PAME biosynthesis in differentiated 3T3-L1 adipocytes (Fig. 3A). Tolcapone, a highly selective COMT inhibitor
(1-1000 nM), also inhibited endogenous PAME and $\left[{ }^{13} \mathrm{C}_{16}\right]$ PAME accumulation in the culture medium of differentiated 3T3-L1 adipocytes (Fig. 3B). In vivo, PVAT is located neighboring the arterial wall and mainly composed of adipocytes as shown in Fig. 4. PAME released from PVAT is an important factor to regulate the vascular tension. $\left[{ }^{13} \mathrm{C}_{16}\right]$-PAME synthesis in Krebs-Henseleit solution following incubation with PVAT isolated from SD rats (Supplemental Fig. 3), WKY rats,
A

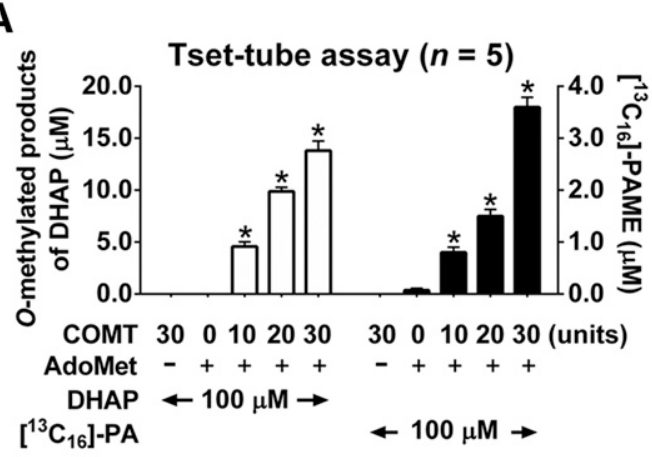

C

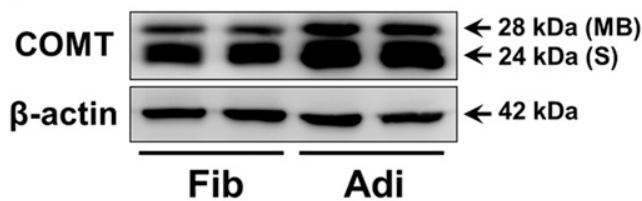

B

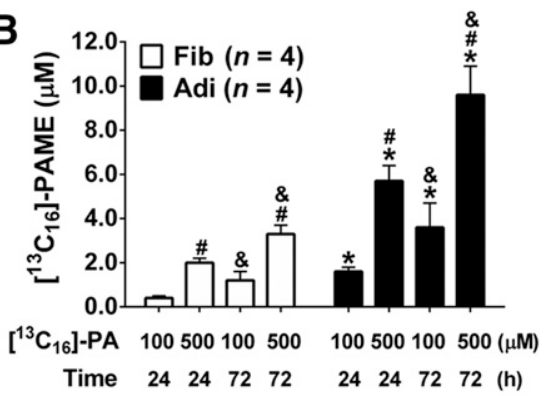

D

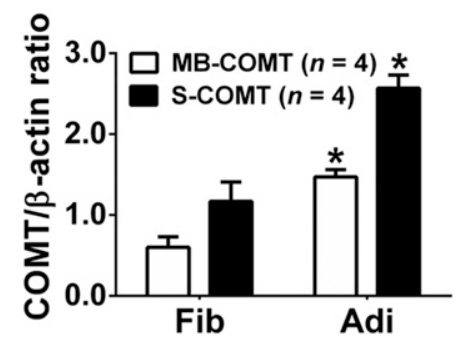

Fig. 2. COMT-catalyzed PAME synthesis. (A) Generation of $O$-methylated products of DHAP (open bars) and $\left[{ }^{13} \mathrm{C}_{16}\right]$-PAME (solid bars) under the condition listed on the plot. The contents of the test tubes are listed on the plot. - and + represent the absence and presence of AdoMet $(1 \mathrm{mM})$ respectively. DHAP or $\left[{ }^{13} \mathrm{C}_{16}\right]-\mathrm{PA}(100 \mu \mathrm{M})$ were separately added to the test tubes, where the COMT concentration was adjusted to $0-30 \mathrm{U} . * P<0.05$ indicates a significant difference from the group without COMT. (B) Effect of 3T3-L1 adipocyte differentiation on $\left[{ }^{13} \mathrm{C}_{16}\right]$-PAME biosynthesis. $\left[{ }^{13} \mathrm{C}_{16}\right]$-PAME concentrations in the media were measured after 24 or 72 hours of culture with 3T3-L1 adipocytes in the fibroblast (Fib) (open bars) or adipocyte (Adi) (solid bars) stage in the presence of 100 or $500 \mu \mathrm{M}\left[{ }^{13} \mathrm{C}_{16}\right]$-PA. Representative Western blots (C) and a summarized bar graph (D) for protein expression of soluble (S)- and membrane-bound (MB)COMT in 3T3-L1 adipocytes at the Fib and Adi stages. ${ }^{*} P<0.05$ vs. COMT-omitted group in (A) and vs. the respective Fib-stage group in (B and D). ${ }^{\#} P<0.05$ vs. groups treated with $100 \mu \mathrm{M}\left[{ }^{13} \mathrm{C}_{16}\right]-\mathrm{PA}$ in (B). ${ }^{\circledR} P<$ 0.05 vs. the respective 24 -hour-culture group in (B). Values are presented as means \pm S.E.M. 
and SHRs (Fig. 5, A and B) was also detected in the presence of $250 \mu \mathrm{M}\left[{ }^{13} \mathrm{C}_{16}\right]$-PA. Increase in $\left[{ }^{13} \mathrm{C}_{16}\right]$-PAME concentration was time-dependent. The peak concentration was reached after 30 minutes of incubation and gradually decreased after 45 minutes of incubation. PVAT preincubation with $300 \mathrm{nM}$ or $1 \mu \mathrm{M}$ tolcapone for 30 minutes decreased $\left[{ }^{13} \mathrm{C}_{16}\right]$-PAME generation, regardless of whether PVAT was isolated from $\mathrm{SD}$ rats, WKY rats, or SHRs.

Losartan Reverses the Decreased PAME Biosynthesis in Established SHR PVAT. More $\left[{ }^{13} \mathrm{C}_{16}\right]$-PAME was synthesized in 13-week-old WKY rats than in 8-week-old WKY rats. Conversely, less $\left[{ }^{13} \mathrm{C}_{16}\right]$-PAME was synthesized from $\left.{ }^{13} \mathrm{C}_{16}\right]$-PA in PVAT of 13 -week-old SHRs than that of 13 -weekold WKY rats (Fig. 5B). In 13-week-old established SHRs, the suppressed $\left[{ }^{13} \mathrm{C}_{16}\right]$-PAME biosynthesis was recovered to normal following the preincubation of PVAT with $1 \mu \mathrm{M}$ losartan $\left(n=5\right.$, Fig. 5B). However, $\left[{ }^{13} \mathrm{C}_{16}\right]$-PAME biosynthesis remained unaffected in 13 -week-old normotensive WKY rats $(n=5)$. Preincubation with $1 \mu \mathrm{M}$ tolcapone decreased $\left[{ }^{13} \mathrm{C}_{16}\right]$ PAME biosynthesis in both 13 -week-old SHRs and WKY rats $(n=5)$.

COMT Expression in PVAT of SHRs and WKY Rats. Similar to 3T3-L1 adipocytes, the PVAT of 8- and 13-week-old SHRs and WKY rats showed MB-COMT and S-COMT expression (Fig. 5, C and D; Supplemental Fig. 5). The 8-week-old prehypertensive SHRs, which showed normal systolic blood

A

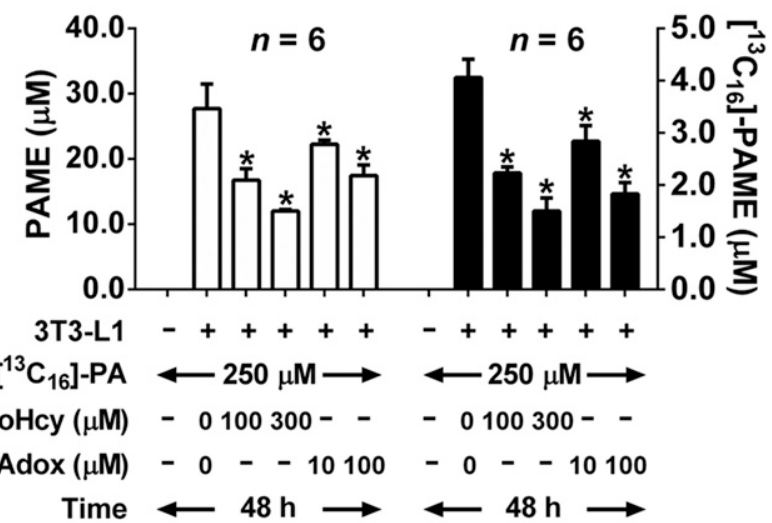

B
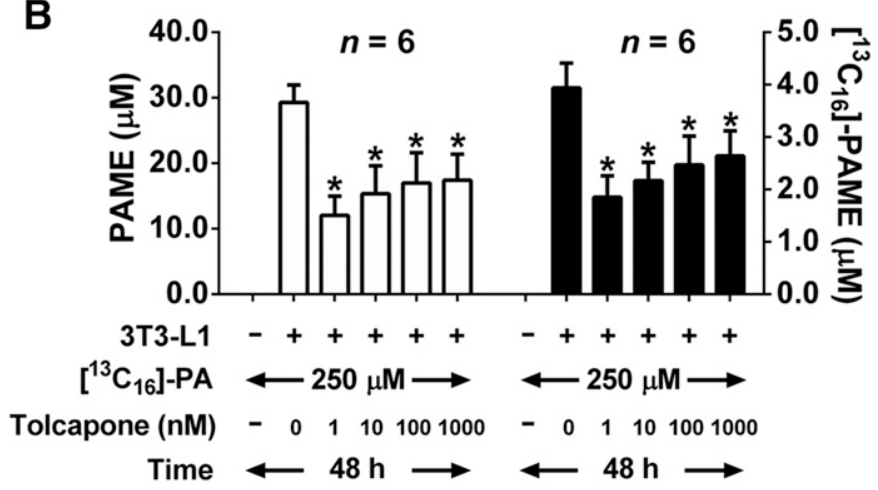

Fig. 3. Suppression of PAME biosynthesis by COMT and AdoHcy hydroxylase inhibitors in 3T3-L1 adipocytes. (A) Differentiated 3T3-L1 adipocytes were incubated in a culture medium containing $\left[{ }^{13} \mathrm{C}_{16}\right]-\mathrm{PA}$ plus AdoHcy (A), Adox (A), or tolcapone (B) for 48 hours. The levels of endogenous PAME (open bars) and $\left[{ }^{13} \mathrm{C}_{16}\right]$-PAME (solid bars) under the conditions indicated on the graph were measured using GC-MS. - and + represent the absence and presence of 3T3-L1 adipocytes or inhibitors, respectively. Values are means \pm S.E.M. $* P<0.05$ vs. the control group.

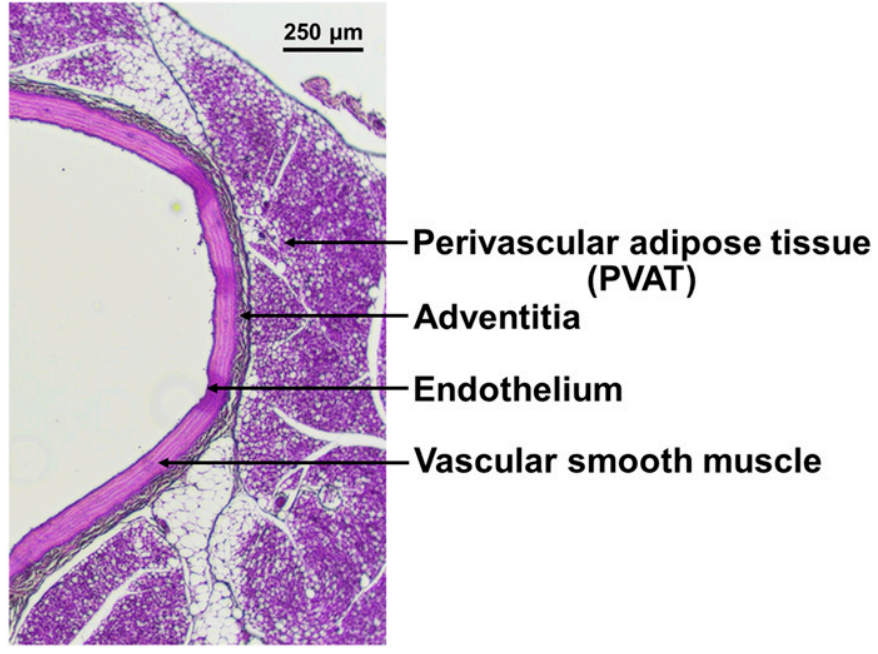

Fig. 4. A cross-sectioned aortic ring showing the relative location of PVAT to the aortic wall. The aortic specimen was taken from a WKY rat and processed with hematoxylin-eosin Y staining. The layers in the aortic ring were labeled on the plot, and enlarged pictures for aortic and mesenteric PVATs were presented in Supplemental Fig. 6. Scale bar, $250 \mu \mathrm{m}$.

pressure compared with age-matched WKY rats (Table 1), showed almost similar MB- and S-COMT expression in aortic PVAT to age-matched WKY rats. In contrast, 13-week-old SHRs, which exhibited significantly higher systolic blood pressure than age-matched WKY rats (Table 1), showed lower MB- and S-COMT expression in aortic PVAT than age-matched controls (Fig. 5, C and D; Supplemental Fig. 5).

COMT-Expressing Adipocytes in PVAT. The existence of COMT enzyme in PVAT were demonstrated by the presence of COMT-immunoreactive adipocytes (Fig. $6 ; n=12$ ). COMT immunofluorescence was localized in the cytoplasmic compartment except for lipid droplet in adipocytes. However, no COMT immunofluorescence was detected when primary or secondary antibodies were excluded during incubation (negative control).

\section{Discussion}

PAME is a PVATRF that drastically decreases in established SHRs (Lee et al., 2011). However, how PAME is synthesized in PVAT remains unclear. In test tube, PAME is synthesized from PA and AdoMet by COMT, an $O$-methylation enzyme (as depicted in Fig. 1C). In cell level, PAME is also generated in 3T3L1 and PVAT adipocytes, which contain COMT enzyme. A schematic diagram (Fig. 7) illustrates PAME biosynthesis in PVAT adipocytes. PAME biosynthesis is inhibited by different COMT inhibitors. In the hypertensive rat model, COMT expression as well as PAME generation in isolated PVAT was lower in established SHRs than in agematched WKY rats.

Computational molecular modeling analysis revealed stable binding of PA to the binding pocket of S-COMT formed by AdoMet and $\mathrm{Mg}^{2+}$, as evidenced by root-mean-square deviation simulations, in which the PA-S-COMT complex was continuously stabilized at the distances (between the oxygen atom in the hydroxyl group of PA and the carbon atom in the methyl group of AdoMet) of 0-200 ns. Interestingly, although PA does not contain a catechol structure, PAME was synthesized by PA methylation via COMT catalysis as simulated in 
A
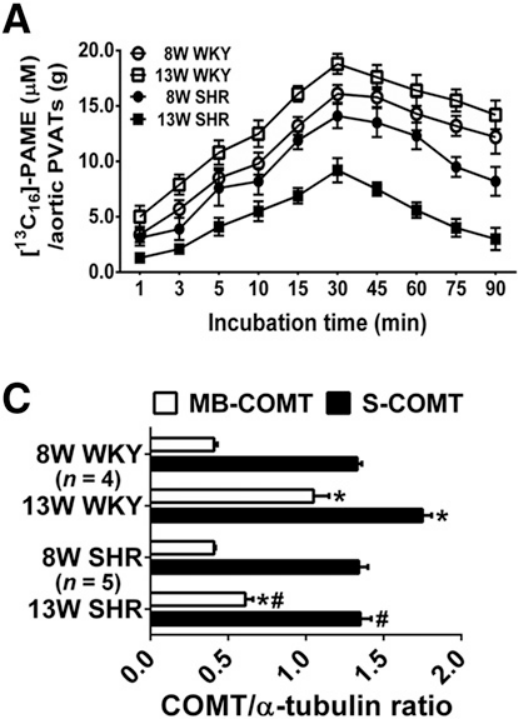

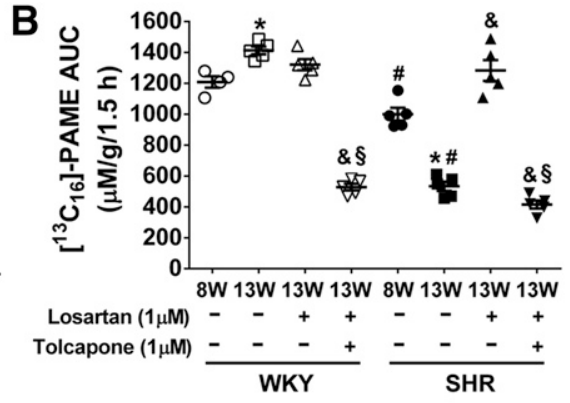

D

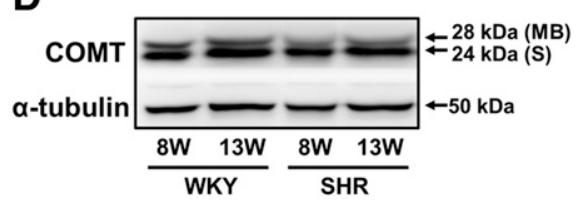

Fig. 5. Decreased COMT expression and PAME biosynthesis in isolated aortic PVATs of SHRs. (A) Time-dependent accumulation of $\left[{ }^{13} \mathrm{C}_{16}\right]$-PAME in Krebs-Henseleit solution containing isolated PVAT obtained from WKY rats and SHRs for 1-90-minute incubation in the presence of $250 \mu \mathrm{M}\left[{ }^{13} \mathrm{C}_{16}\right]-\mathrm{PA}$. (B) The area under each curve (AUC), which represents cumulative $\left[{ }^{13} \mathrm{C}_{16}\right]$-PAME generation, under the different drug pretreatment was summarized. Ages and respective marks are indicated on the plot. - and + represent the absence and presence of $1 \mu \mathrm{M}$ losatran or tolcapone, respectively. (C and $\mathrm{D})$ Membrane-bound (MB)- and soluble (S)-COMT expression in aortic PVAT of 8- or 13 -week-old SHRs and WKY rats ( $n=4$ to 5 ). $\alpha$-Tubulin levels were served as the loading control. Values are presented as means \pm S.E.M. ${ }^{*} P<0.05$ indicates significant difference from the 8-week-old group without treatment. ${ }^{\#} P<0.05$ indicates significant difference from age-matched WKY rats. ${ }^{\circledR} P<0.05$ indicates significant difference from the age-matched group without losartan treatment. ${ }^{\S} P<0.05$ indicates significant difference from the age-matched group without tolcapone treatment. computational molecular modeling and as demonstrated in in vitro enzymatic assays and pharmacological inhibitor experiments. Typically, PA is one of the most common saturated fatty acids found in the human body and can be obtained through diet and endogenous synthesis via de novo lipogenesis or lipolysis from triacylglycerol (TG) in adipose tissue. However, excess endogenous PA might induce endoplasmic reticulum stress (Tse et al., 2018) and lipotoxicity in various cell types (Peng et al., 2011). It has been believed that degradation, desaturation, and elongation of PA might be protective and regulated by homeostasis mechanisms to prevent cells from lipid-related stress-induced damage (Green and Olson, 2011). In addition, methylation might be an alternative means to reduce PA-induced stress in the human body.

Endogenous fatty acid methyl esters (FAMEs), including PAME, are synthesized in various animal tissues, such as the

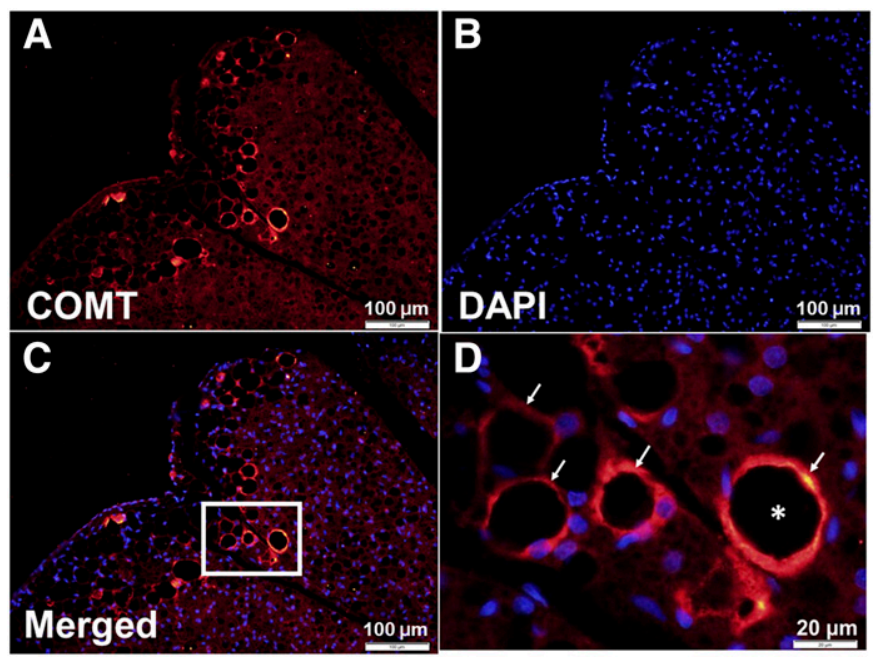

Fig. 6. COMT immunoreactivity in PVAT adipocytes. (A and B) Representative images showing an aortic section immunostained with COMT and DAPI. (C) A merged image indicating the location of COMT enzyme and nuclei. The area in the red box was enlarged and presented in (D). Arrows indicate COMT-immunoreactive adipocytes, and a star indicates the presence of lipid droplet. DAPI, 4',6-diamidino-2-phenylindole. lung (Zatz et al., 1981), pancreas (Leikola et al., 1965; Lough and Garton, 1968), retinas, and parotid (Kloog et al., 1982). However, the precursors and/or exact methyl transferases involved remain unknown. Previous studies on a rat model (Kaphalia et al., 1995) and human hepatoma HepG2 cells (Kaphalia et al., 1999) have demonstrated that endogenous FAMEs are synthesized following exposure to methanol, suggesting that carboxylesterase (EC 3.1.1.1) is a FAME methyltransferase. However, carboxylesterase is an AdoMetindependent enzyme, and its methylation usually requires external methanol. Therefore, to avoid the additional impact of methanolic solvents, we did not use methanol in our incubation experiments for PAME biosynthesis.

COMT is implicated in hypertension and cardiovascular diseases (Houston, 2007). COMT expression and activity decrease in hypertensive patients, resulting in elevated circulating epinephrine and norepinephrine levels (Hirano et al., 2007) and depleted 2-methoxyestradiol (a vasodilator) levels (Hernandez et al., 2013). The decreased COMT level further increases systemic arterial pressure. Previous studies have reported that COMT expression and activity are reduced in the liver (Tsunoda et al., 2003), brain (Masuda et al., 2006), and renal cortex (Ooshima et al., 2009) in SHRs. MB- and $\mathrm{S}-\mathrm{COMT}$ expression was disproportionate in the aortic PVAT of established SHRs in parallel with PAME decrease. In addition, the plasma levels of TG and nonesterified free fatty acids, which are resources for PAME biosynthesis, were lower in 3-month-old male SHRs than in age-matched male WKY rats (Gálvez et al., 2006). Consequently, decreased COMT expression in PVAT and fat contents in adipocytes might contribute to inhibition of PAME biosynthesis, and this might be a relevant factor in hypertension pathogenesis.

PVAT dysfunction in the hypertensive state is characterized by the loss of the anticontractile activity of PVAT (Lee et al., 2011). PVAT, which plays a paracrine role in regulating vasomotor function, releases vasodilators or vasoconstrictors (e.g., Ang II). Ang II exists (Gálvez-Prieto et al., 2008) and increases in PVAT of established SHRs (Lu et al., 2010; Lee et al., 2011). Therefore, in the hypertensive state, Ang II synthesis increases and PAME biosynthesis or release decreases in PVAT, leading to decreased arterial smooth 


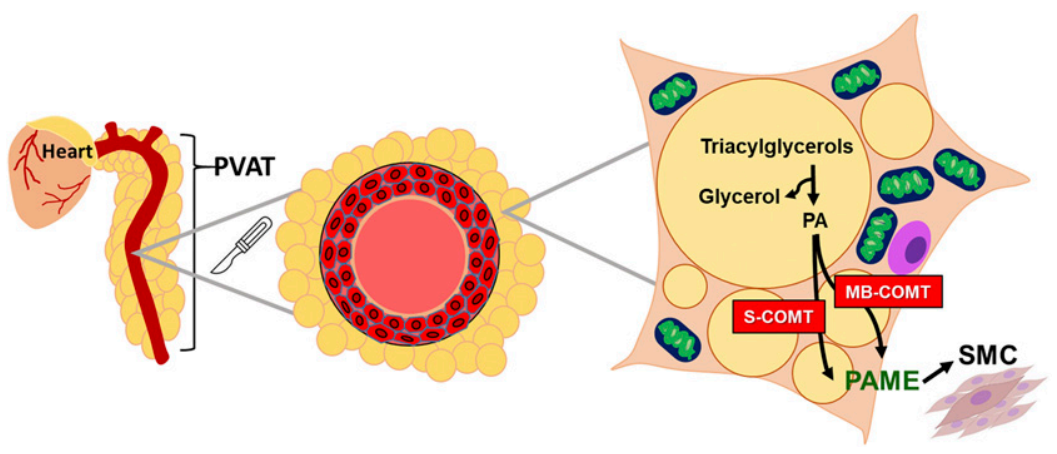

Fig. 7. A schematic diagram depicting PAME biosynthesis in PVAT adipocytes. PA is produced from the lipolysis of triacylglycerol in PVAT adipocytes. PA is subsequently $O$-methylated to form PAME by MB- or S-COMT. After released from PVAT, PAME may modulate the vascular smooth muscle activity. SMC, smooth muscle cell.

muscle relaxation (Lee et al., 2011; Wang et al., 2018). Losartan is an Ang II receptor type 1 (AT-1) receptor antagonist used to treat hypertension. Previous studies have reported that losartan treatment reverses decreased PAME release from PVAT in established SHRs (Lee et al., 2011). In addition, the Ang II pathway inhibits TG lipolysis, which is also reversed by losartan treatment (Cabassi et al., 2005). Losartan can reverse low PAME biosynthesis levels in PVAT of established SHRs, indicating the involvement of PAME in losartan-induced antihypertension effects.

There are several limitations in this study. First, the acquisition of human tissue is restricted. Therefore, we could not study PAME biosynthesis and COMT expression in human PVAT adipocytes. Second, there is no specific antibody or inhibitor that can separate the effects of MB- from S-COMT on PAME biosynthesis. Thus, we could not determine which of the two, MB-COMT or S-COMT, is a major factor in PAME biosynthesis in PVAT.

In conclusion, we, for the first time, demonstrate that PAME is synthesized through PA methylation via the AdoMetdependent COMT catalytic pathway in adipocytes (depicted in Fig. 7). In addition, the reversal of decreased PAME generation in PVAT by losartan in hypertensive rats indicates the involvement of AT-1 receptor in PAME synthesis. The role of COMT-dependent PAME biosynthesis in AT-1 receptor-mediated blood pressure regulation remains to be determined. However, because of the vasodilative effect of PAME illustrated by Lee et al. (2011), targeting the PAME biosynthetic pathway in PVAT might help in developing therapeutic or preventive strategies against hypertension.

\section{Acknowledgments}

We thank the laboratory member of Dr. Jih-I Yeh for assistance in culturing the 3T3-L1 cell line and Dr. Jon-Son Kuo for critical reading of the manuscript.

\section{Authorship Contributions}

Participated in research design: Liu, Chen, Lee.

Conducted experiments: Liu, Tseng, Lin, Weng.

Performed data analysis: Liu, Hsu, Weng.

Wrote or contributed to the writing of the manuscript: Liu, Chen, Hsu, Lee.

\section{References}

Bartel RL and Borchardt RT(1984) Effects of adenosine dialdehyde on S-adenosylhomocysteine hydrolase and S-adenosylmethionine-dependent transmethylations in mouse L929 cells. Mol Pharmacol 25:418-424.

Borchardt RT and Huber JA(1975) Catechol O-methyltransferase. 5. Structureactivity relationships for inhibition by flavonoids. $J$ Med Chem 18:120-122.

Cabassi A, Coghi P, Govoni P, Barouhiel E, Speroni E, Cavazzini S, Cantoni AM, Scandroglio R, and Fiaccadori E(2005) Sympathetic modulation by carvedilol and losartan reduces angiotensin II-mediated lipolysis in subcutaneous and visceral fat. J Clin Endocrinol Metab 90:2888-2897.

Chang CC, Hsu HJ, Yen JH, Lo SY, and Liou JW(2017) A Sequence in the loop domain of hepatitis $\mathrm{C}$ virus $\mathrm{E} 2$ protein identified in silico as crucial for the selective binding to human CD81. PLoS One 12:e177383.

Chang L, Villacorta L, Li R, Hamblin M, Xu W, Dou C, Zhang J, Wu J, Zeng R, and Chen YE(2012) Loss of perivascular adipose tissue on peroxisome proliferator-activated receptor- $\gamma$ deletion in smooth muscle cells impairs intravascular thermoregulation and enhances atherosclerosis. Circulation 126 1067-1078.

Chou CL, Pang CY, Lee TJ, and Fang TC(2013) Direct renin inhibitor prevents and ameliorates insulin resistance, aortic endothelial dysfunction and vascular remodeling in fructose-fed hypertensive rats. Hypertens Res 36:123-128.

Eringa EC, Bakker W, and van Hinsbergh VW(2012) Paracrine regulation of vascular tone, inflammation and insulin sensitivity by perivascular adipose tissue. Vascul Pharmacol 56:204-209.

Fésüs G, Dubrovska G, Gorzelniak K, Kluge R, Huang Y, Luft FC, and Gollasch $\mathrm{M}(2007)$ Adiponectin is a novel humoral vasodilator. Cardiovasc Res 75:719-727.

Gálvez B, de Castro J, Herold D, Dubrovska G, Arribas S, González MC, Aranguez I, Luft FC, Ramos MP, Gollasch M, et al.(2006) Perivascular adipose tissue and mesenteric vascular function in spontaneously hypertensive rats. Arterioscler Thromb Vasc Biol 26:1297-1302.

Gálvez-Prieto B, Bolbrinker J, Stucchi P, de Las Heras AI, Merino B, Arribas S, RuizGayo M, Huber M, Wehland M, Kreutz R, et al.(2008) Comparative expression analysis of the renin-angiotensin system components between white and brown perivascular adipose tissue. J Endocrinol 197:55-64.

Gao YJ, Lu C, Su LY, Sharma AM, and Lee RM(2007) Modulation of vascular function by perivascular adipose tissue: the role of endothelium and hydrogen peroxide. Br J Pharmacol 151:323-331.

Green CD and Olson LK(2011) Modulation of palmitate-induced endoplasmic reticulum stress and apoptosis in pancreatic $\beta$-cells by stearoyl-CoA desaturase and Elovl6. Am J Physiol Endocrinol Metab 300:E640-E649.

Hernandez M, Hernandez I, Rodriguez F, Pertegal M, Bonacasa B, Salom MG, Quesada T, and Fenoy FJ(2013) Endothelial dysfunction in gestational hypertension induced by catechol-O-methyltransferase inhibition. Exp Physiol 98: 856-866.

Hirano Y, Tsunoda M, Shimosawa T, Matsui H, Fujita T, and Funatsu T(2007) Suppression of catechol-O-methyltransferase activity through blunting of alpha2adrenoceptor can explain hypertension in Dahl salt-sensitive rats. Hypertens Res 30:269-278.

Hou T, Wang J, Li Y, and Wang W(2011a) Assessing the performance of the MM/ PBSA and MM/GBSA methods. 1. The accuracy of binding free energy calculations based on molecular dynamics simulations. J Chem Inf Model 51:69-82.

Hou T, Wang J, Li Y, and Wang W(2011b) Assessing the performance of the molecular mechanics/Poisson Boltzmann surface area and molecular mechanics/generalized Born surface area methods. II. The accuracy of ranking poses generated from docking. J Comput Chem 32:866-877.

Hou T and Yu R(2007) Molecular dynamics and free energy studies on the wild-type and double mutant HIV-1 protease complexed with amprenavir and two amprenavir-related inhibitors: mechanism for binding and drug resistance. $J$ Med Chem 50:1177-1188.

Houston MC(2007) The role of mercury and cadmium heavy metals in vascular disease, hypertension, coronary heart disease, and myocardial infarction. Altern Ther Health Med 13:S128-S133.

Htun NC, Miyaki K, Song Y, Ikeda S, Shimbo T, and Muramatsu M(2011) Association of the catechol-O-methyl transferase gene Val158Met polymorphism with blood pressure and prevalence of hypertension: interaction with dietary energy intake. Am J Hypertens 24:1022-1026.

Jiang SJ, Liou JW, Chang CC, Chung Y, Lin LF, and Hsu HJ(2015) Peptides derived from CXCL8 based on in silico analysis inhibit CXCL8 interactions with its receptor CXCR1. Sci Rep 5:18638.

Kanasaki K, Palmsten K, Sugimoto H, Ahmad S, Hamano Y, Xie L, Parry S, Augustin HG, Gattone VH, Folkman J, et al.(2008) Deficiency in catechol-O-methyltransferase and 2-methoxyoestradiol is associated with pre-eclampsia. Nature 453: 1117-1121.

Kaphalia BS, Carr JB, and Ansari GA(1995) Increased endobiotic fatty acid methyl esters following exposure to methanol. Fundam Appl Toxicol 28:264-273.

Kaphalia BS, Green SM, and Ansari GA(1999) Fatty acid ethyl and methyl ester synthases, and fatty acid anilide synthase in HepG2 and AR42J cells: interrelationships and inhibition by tri-o-tolyl phosphate. Toxicol Appl Pharmacol 159: 134-141. 
Kawai M, Namba N, Mushiake S, Etani Y, Nishimura R, Makishima M, and Ozono $\mathrm{K}(2007)$ Growth hormone stimulates adipogenesis of 3T3-L1 cells through activation of the Stat5A/5B-PPARgamma pathway. J Mol Endocrinol 38:19-34

Kloog Y, Zatz M, Rivnay B, Dudley PA, and Markey SP(1982) Nonpolar lipid methylation-identification of nonpolar methylated products synthesized by rat basophilic leukemia cells, retina and parotid. Biochem Pharmacol 31:753-759.

Kurkela M, Siiskonen A, Finel M, Tammela P, Taskinen J, and Vuorela P(2004) Microplate screening assay to identify inhibitors of human catechol-O-methyltransferase. Anal Biochem 331:198-200.

Lee YC, Chang HH, Chiang CL, Liu CH, Yeh JI, Chen MF, Chen PY, Kuo JS, and Lee TJ(2011) Role of perivascular adipose tissue-derived methyl palmitate in vascular tone regulation and pathogenesis of hypertension. Circulation 124:1160-1171.

Lee YC, Chang HH, Liu CH, Chen MF, Chen PY, Kuo JS, and Lee TJ(2010) Methyl palmitate: a potent vasodilator released in the retina. Invest Ophthalmol Vis Sci 51:4746-4753.

Lee RH, Couto E Silva A, Possoit HE, Lerner FM, Chen PY, Azizbayeva R, Citadin CT, Wu CY, Neumann JT, and Lin HW(2019) Palmitic acid methyl ester is a novel neuroprotective agent against cardiac arrest. Prostaglandins Leukot Essent Fatty Acids 147:6-14.

Leikola E, Nieminen E, and Salomaa E(1965) Occurrence of methyl esters in the pancreas. J Lipid Res 6:490-493.

Lin HW, Liu CZ, Cao D, Chen PY, Chen MF, Lin SZ, Mozayan M, Chen AF, Premkumar LS, Torry DS, et al.(2008) Endogenous methyl palmitate modulates nicotinic receptor-mediated transmission in the superior cervical ganglion. Proc Natl Acad Sci USA 105:19526-19531.

Liou JW, Chang FT, Chung Y, Chen WY, Fischer WB, and Hsu HJ(2014) In silico analysis reveals sequential interactions and protein conformational changes during the binding of chemokine CXCL-8 to its receptor CXCR1. PLoS One 9:e94178.

Lough AK and Garton GA(1968) The lipids of human pancreas with special reference to the presence of fatty acid methyl esters. Lipids 3:321-323.

$\mathrm{Lu} \mathrm{C}$, Su LY, Lee RM, and Gao YJ(2010) Mechanisms for perivascular adipose tissuemediated potentiation of vascular contraction to perivascular neuronal stimulation: the role of adipocyte-derived angiotensin II. Eur J Pharmacol 634:107-112.

Ma Z, Liu H, and Wu B(2014) Structure-based drug design of catechol-O-methyltransferase inhibitors for CNS disorders. Br J Clin Pharmacol 77:410-420.

Männistö PT and Kaakkola S(1999) Catechol-O-methyltransferase (COMT): biochemistry, molecular biology, pharmacology, and clinical efficacy of the new selective COMT inhibitors. Pharmacol Rev 51:593-628.

Masuda M, Tsunoda M, and Imai K(2006) Low catechol-O-methyltransferase activity in the brain and blood pressure regulation. Biol Pharm Bull 29:202-205.

Ooshima K, Ozaki S, Tabuchi M, Higashino H, Honda E, Park AM, Arima S, and Munakata $\mathrm{H}(2009)$ Decreased expression of catechol-O-methyltransferase in the renal cortex of malignant spontaneously hypertensive rats. Tohoku J Exp Med 219:331-336.

Peng G, Li L, Liu Y, Pu J, Zhang S, Yu J, Zhao J, and Liu P(2011) Oleate blocks palmitate-induced abnormal lipid distribution, endoplasmic reticulum expansion and stress, and insulin resistance in skeletal muscle. Endocrinology 152: $2206-2218$

Schüttelkopf AW and van Aalten DM(2004) PRODRG: a tool for high-throughput crystallography of protein-ligand complexes. Acta Crystallogr D Biol Crystallogr 60:1355-1363

Song W, Luo Q, Zhang Y, Zhou L, Liu Y, Ma Z, Guo J, Huang Y, Cheng L, Meng Z, et al.(2019) Organic cation transporter 3 (Oct3) is a distinct catecholamines clearance route in adipocytes mediating the beiging of white adipose tissue. PLoS Biol 17:e2006571.

Stanley JL, Andersson IJ, Poudel R, Rueda-Clausen CF, Sibley CP, Davidge ST, and Baker PN(2012) Sildenafil citrate rescues fetal growth in the catechol-Omethyl transferase knockout mouse model. Hypertension 59:1021-1028.

Stewart SH, Oroszi G, Randall PK, and Anton RF(2009) COMT genotype influences the effect of alcohol on blood pressure: results from the COMBINE study. Am J Hypertens 22:87-91.

Szasz T, Bomfim GF, and Webb RC(2013) The influence of perivascular adipose tissue on vascular homeostasis. Vasc Health Risk Manag 9:105-116.

Tano JY, Schleifenbaum J, and Gollasch M(2014) Perivascular adipose tissue, potassium channels, and vascular dysfunction. Arterioscler Thromb Vasc Biol 34: $1827-1830$

Tehlivets $\mathrm{O}(2011)$ Homocysteine as a risk factor for atherosclerosis: is its conversion to s-adenosyl-L-homocysteine the key to deregulated lipid metabolism? J Lipids 2011:702853.

Tse EK, Salehi A, Clemenzi MN, and Belsham DD(2018) Role of the saturated fatty acid palmitate in the interconnected hypothalamic control of energy homeostasis and biological rhythms. Am J Physiol Endocrinol Metab 315:E133-E140.

Tseng TL, Chen MF, Liu CH, Pang CY, Hsu YH, and Lee TJ(2016) Induction of endothelium-dependent constriction of mesenteric arteries in endotoxemic hypotensive shock. Br J Pharmacol 173:1179-1195.

Tseng TL, Chen MF, Tsai MJ, Hsu YH, Chen CP, and Lee TJ(2012) Oroxylin-A rescues LPS-induced acute lung injury via regulation of $\mathrm{NF}-\kappa \mathrm{B}$ signaling pathway in rodents. PLoS One 7:e47403.

Tsunoda M, Tenhunen J, Tilgmann C, Arai H, and Imai K(2003) Reduced membranebound catechol-O-methyltransferase in the liver of spontaneously hypertensive rats. Hypertens Res 26:923-927.

Vieira-Coelho MA and Soares-da-Silva P(1999) Effects of tolcapone upon soluble and membrane-bound brain and liver catechol-O-methyltransferase. Brain Res 821 $69-78$.

Wang N, Kuczmanski A, Dubrovska G, and Gollasch M(2018) Palmitic acid methyl ester and its relation to control of tone of human visceral arteries and rat aortas by perivascular adipose tissue. Front Physiol 9:583.

Wójcicka G, Jamroz-Wiśniewska A, Atanasova P, Chaldakov GN, Chylińska-Kula B and Bełtowski J(2011) Differential effects of statins on endogenous H2S formation in perivascular adipose tissue. Pharmacol Res 63:68-76.

Xu A, Wang Y, Lam KS, and Vanhoutte PM(2010) Vascular actions of adipokines molecular mechanisms and therapeutic implications. Adv Pharmacol 60:229-255.

Xu L, Li Y, Sun H, Li D, and Hou T(2013) Structural basis of the interactions between CXCR4 and CXCL12/SDF-1 revealed by theoretical approaches. Mol Biosyst 9: $2107-2117$

Yeh JC, Borchardt RT, and Vedani A(1991) A molecular model for the active site of S-adenosyl-L-homocysteine hydrolase. J Comput Aided Mol Des 5:213-234.

Zatz M, Dudley PA, Kloog Y, and Markey SP(1981) Nonpolar lipid methylation. Biosynthesis of fatty acid methyl esters by rat lung membranes using S-adenosylmethionine. J Biol Chem 256:10028-10032.

Address correspondence to: Mei-Fang Chen, Department of Medical Research, Hualien Tzu Chi Hospital, 707, Sec 3, Chung Yang Road, Hualien, Taiwan 970. E-mail: mfchen@mail.tcu.edu.tw 